\title{
A COMPUTER SIMULATION STUDY OF IONIC CONDUCTIVITY IN POLYMER ELECTROLYTES
}

\author{
Aninda Jiban Bhattacharyya円, T.R.Middya and S.Tarafdar \\ Condensed Matter Physics Research Center, Department of Physics, \\ Jadavpur University, Calcutta-700032, INDIA
}

Pacs No. : 66.30.Dn; 61.43.Bn; 61.41.+e

Keywords : polymer electrolytes, ionic conductivity, random walk, computer simulation

\begin{abstract}
In this paper we present a computer simulation study of ionic conductivity in solid polymeric electrolytes. The multiphase nature of the material is taken into account. The polymer is represented by a regular lattice whose sites represent either crystalline or amorphous regions with the charge carrier performing a random walk. Different waiting times are assigned to sites corresponding to the different phases. A random walk (RW) is used to calculate the conductivity through the Nernst-Einstein relation. Our walk algorithm takes into account the reorganisation of the different phases over time scales comparable to time scales for the conduction process. This is a characteristic feature of the polymer network. The qualitative nature of the variation of conductivity with salt concentration agrees with the experimental values for $\mathrm{PEO}-\mathrm{NH}_{4} \mathrm{I}$ and $\mathrm{PEO}-\mathrm{NH}_{4} \mathrm{SCN}$. The average jump distance estimated from our work is consistent with the reported bond lengths for such polymers.
\end{abstract}

\footnotetext{
1 e-mail:aninda@juphys.ernet.in
} 


\section{Introduction}

During the last two decades a lot of interest has been generated in the potential industrial applications of polymer electrolytes [1,2]. Hence, developement of theoretical understanding of such materials is essential. Polymeric solid electrolytes are formed by complexing an ionic salt like $\mathrm{NaI}, \mathrm{NH}_{4} \mathrm{SCN}$, etc with polymers, for example polyethylene oxide (PEO), polypropylene oxide (PPO), [3,4] etc. We restrict our discussion to solvent free polymer electrolytes which are formed by dissolving or suspending the salt and the polymer in a suitable solvent and then evaporating the solvent during casting.

The theoretical explanation of the dependence of ionic transport on temperature, salt fraction, frequency, etc. is a formidable problem. The complications arise mainly because these polymer systems are multiphase in nature. Further difficulties creep in because the presence of different phases are dependent on processing conditions and thermal history.

The aim of this work is to study the variation of ionic conductivity in a polymer electrolyte where the salt fraction is varied. The varying salt fraction changes the proportion of crystalline and amorphous regions and also the concentration of charge carriers supplied by the salt [5].

The final goal is of course to be able to predict the conductivity of an unknown polymer complex with a given salt fraction. In this work we have not fully achieved this since we have to use the experimental data for crystallinity at different salt fractions as input. We first briefly describe previous work done in this field and then present our model.

Since analytical calculation of conductivity in such a complex system is very difficult, a class of models frequently used to study conductivity in disordered media involve computer simulation of a random walk (RW) [6-8]. Appropriate algorithms to represent phases of different conductivity may be incorporated into such models. Often a continuous time random walk (CTRW) [8] is used. A recent work [9] introduces a method for speeding up a CTRW calculation by a modified blind ant algorithm which makes the walker jump at every step but adjusts the time to account for the waiting time.

The dynamic bond percolation model by Druger et al $[10,11]$ is used to calculate the conductivity of a polymer through computer simulation of a hopping model. This model incorporates the dynamic nature of the medium by introducing a renewal time. The frequency dependence 
of the conductivity within a certain range can be explained by this model. The model fails however at the very high frequency limit, since inertial effects are not taken into account.

\section{The Model}

In the present paper our main aim is to develop an algorithm utilising some experimental inputs to explain the salt fraction dependence of ionic conductivity of polymer-salt systems. The solvent free polymer electrolytes are prepared in the form of thin films, so as a preliminary approximation, in this paper we consider the polymer electrolyte as a two dimensional space lattice. Rather than dealing with bonds, our model deals with sites only. A random walk is executed on the square lattice. The uniform square lattice does not of course represent the structure of the polymer network which is disordered, but only provides a convenient space for executing the random walk.

We designate a site on the lattice as amorphous or crystalline. The sites actually represent very small regions of the polymer complex which belong to a single phase only, and the lattice spacing $(\xi)$ represents the distance between such sites. We assign a jump probability to each site representing the conductivity of the phase to which the site belongs.

However, our model is not a "quenched" model. The walker sees a site as crystalline or amorphous statistically according to a random number chosen on its visit to the site. The probability of the site being, say crystalline, is determined from the experimental crystallinity. This means that a site previously found to be crystalline may become amorphous on a later visit. A crystalline site may also become amorphous or vice versa, during the waiting time of the walker at that site.

This procedure is often adopted to make the walk algorithm simpler and provide an effectively infinite system. Here however, it gives an added advantage. The polymer, as distinct from crystalline or glassy superionic conductors is known to show a dynamic disorder, i.e. the matrix is continually rearranging itself after a characteristic (renewal) time $\tau_{r}$. The RW algorithm thus gives a more realistic picture of the polymer, though in the present formalism we have no control over $\tau_{r}$. We are at present working in a variation of the model where $\tau_{r}$ can be varied, this is to be reported shortly [12]. 


\subsection{Random Walk Algorithm}

The polymer-electrolyte system is multiphase in nature consisting of both crystalline (belonging to complexed and uncomplexed polymer) $[1,3,4]$ and amorphous regions. For simulation at a particular salt fraction, the ratio of amorphous to crystalline sites is taken from experimental estimates of crystallinity from differential thermal analysis (DTA) or x-ray diffraction (XRD), and the distribution of these phases in the lattice is random. A site belonging to the $i^{t h}$ phase is assigned a jump probability $p_{i}$ for jumping to a nearest neighbour site, at each time step. The time elapsed (inverse of $\mathrm{p}_{i}$ ) between arrival at one site and arrival at the next site includes $\tau_{j}$ the time taken to jump from one site to a nearest neighbour and an average waiting time $i \tau_{i} \dot{i}$ at the $\mathrm{i}^{\text {th }}$ site. A longer waiting time corresponds to a lower conductivity of the phase and hence of the site. In the present case we have two waiting times, $\tau_{a}$ for the amorphous phase and $\tau_{c}$ for the crystalline phase which is larger.

A distribution of energetically different sites on a lattice is usually represented in a simulation model by either of the following pictures :

(a) A well model, where each site is a potential well. Here the well depth $\mathrm{w}_{i}$ is characteristic of that particular site i, and determines how long the random walker will be trapped there.

(b) A barrier model where a barrier of height $h_{i j}$, is envisaged between the sites $\mathrm{i}$ and $\mathrm{j}$. Here the probability of hopping to i from $\mathrm{j}$ may be determined by the nature of both the sites $\mathrm{i}$ and j.

In the present work we employ the considerably simpler well model, where the probability of leaving a site is determined by the phase of this site, but the carrier has an equal probability of going to all four nearest neighbours whether they are crystalline or amorphous. In view of the dynamic disorder, where the polymer chains can reorient within the time interval $\tau$, which the random walker takes to hop to a neighbouring site, model (a) seems quite adequate.

In case of normal diffusion, the diffusion coefficient can be obtained from a random walk, through the relation

$$
<r^{2}(t)>=2 d D t
$$

where $\left\langle r^{2}>\right.$ is the average square distance covered by the walker in $t$ time steps and $\mathrm{d}$ is the dimension of the space lattice. The constant D is the diffusion coefficient. 
In real units the diffusion coefficient is given by

$$
D=\frac{<r^{2}>}{4 t} \frac{(\text { distance unit })^{2}}{(\text { time unit })}
$$

The distance unit $=$ the lattice constant $(\xi)$ and time unit $=\tau$, the time step. In our model $\tau$ $=\tau_{j}$ the time taken in jumping from one site to another, waiting time at a site is measured in units of $\tau$. The steps of the RW are given below

1. The walker is released at a randomly chosen site on a two dimensional lattice.

2. A random number $R_{1}$ is chosen. If $R_{1} i c$ (c is the crystallinity), the site is crystalline, otherwise it is amorphous.

3. Now a second random number $R_{2}$ is chosen. The residence probability of the appropriate site is $\left(1-\mathrm{p}_{i}\right)$. So the probability of jumping to a particular neighbour is $\mathrm{p}_{i} / 4$ If

$$
0 \leq R_{2} \leq \frac{p_{i}}{4}
$$

the walker jumps to the left neighbour.

If

$$
\frac{p_{i}}{4}<R_{2} \leq \frac{p_{i}}{2}
$$

the walker jumps to the right neighbour.

If

$$
\frac{p_{i}}{2}<R_{2} \leq \frac{3 p_{i}}{4}
$$

the walker moves to the upper neighbour

and if

$$
\frac{3 p_{i}}{4}<R_{2} \leq p_{i}
$$

the walker moves to the lower neighbour.

If

$$
p_{i}<R_{2} \leq 1
$$

it does not jump at all. 
In case the walker has jumped, it again checks whether the new site is crystalline or amorphous by $\operatorname{step}(2)$. Even if it has not jumped $\operatorname{step}(2)$ is repeated to account for the reorganisation of the lattice i.e. an amorphous site may become crystalline after each time step.

The walk proceeds in this manner for the requisite number of steps. Due to the stochastic nature of the process, one has to average over a large number of such walks to get a meaningful value of $\mathrm{j}^{2} \dot{i}$. In this work the walker does a random walk of (15000-75000) steps and distance (r) covered is averaged over (20000-100000) walks. This gives sufficiently good convergence (up to 3 significant figures) for the diffusion coefficient.

Our random walk algorithm allows the walker to move on an effectively infinite sample. This is possible because here we do not take a quenched system with sites assigned specifically to a definite phase. So the problem of finite size effects is avoided to some extent. But, of course there is a limitation to the size of the walk due to restricted computer time. For normal diffusion the relation between the diffusion coefficient and the conductivity is given by the Nernst-Einstein equation

$$
\sigma=\frac{D N q^{2}}{k T}
$$

where $\mathrm{N}$ is the number density of the mobile ion, $\mathrm{q}$ is the charge of the mobile ion, $k$ is the Boltzmann's constant and $\mathrm{T}$ is the temperature. The above equation is written as

$$
\sigma=K X D
$$

where $\mathrm{K}$ is a constant and $\mathrm{X}$ is the salt fraction. Since in eqn.(3) $k, \mathrm{q}$ and $\mathrm{T}$ are constants for conductivity measured at different salt fractions we have conveniently grouped them into a single constant K. In ion conducting polymers, mobile charge carriers are provided by the salt, the pure polymer being an insulator. So $\mathrm{N}$ which is the number density of charge carriers is assumed to be proportional to the salt fraction $\mathrm{X}$ in eqn.(3).

\section{Waiting times and jump distance in terms of the sim- ulation parameters}

A random walk is usually performed with the time step and lattice constant chosen to be unity for convenience. This will give D in terms of arbitrary units. To compare the value obtained 
with an experimental result, we must assign real values to $\tau$ and $\xi$. This section identifies these quantities in terms of experimentally measurable properties.

Our model lattice contains two types of sites, crystalline (c) and amorphous (a). The relative number of each depends on the salt fraction. Suppose $\tau$ is the time unit for the walk. Let the average waiting time for the amorphous and crystalline sites be $i \tau_{a} \dot{i}$ and $i \tau_{c} \dot{i}$ respectively. Let $\tau_{j}$ be the jump time between sites. Total time $\mathrm{t}$ for the walk is given by

$$
t=N_{c}<\tau_{c}+\tau_{j}>+N_{a}<\tau_{a}+\tau_{j}>
$$

We assume here $\tau_{j}=\tau$ which is a constant. So eqn.(5) becomes,

$$
t=N_{c}\left(t_{c}+1\right) \tau+N_{a}\left(t_{a}+1\right) \tau
$$

where $N_{c}$ and $N_{a}$ are respectively the total number of crystalline and amorphous sites visited during the walk. $\mathrm{t}_{c}$ and $\mathrm{t}_{a}$ are the waiting times measured in units of the jump time $\tau_{j}(=\tau)$. Eqn (6) can be written as

$$
t=\tau\left(t_{a}+1\right)\left[N_{a}+N_{c} \frac{\left(t_{c}+1\right)}{\left(t_{a}+1\right)}\right]
$$

Now $\left(t_{c}+1\right)$ and $\left(t_{a}+1\right)$ are nothing but inverse of the probability for a walker to jump from crystalline and amorphous sites respectively.

$$
t_{c}+1=\frac{1}{p_{c}}: t_{a}+1=\frac{1}{p_{a}}
$$

where $\mathrm{p}_{c}$ and $\mathrm{p}_{a}$ are the jumping probabilities from crystalline and amorphous sites respectively. $\mathrm{p}_{i}$ may vary from 0 to 1 , corresponding to the lowest and highest possible conductivities. For $\mathrm{p}_{i}=0, \mathrm{t}_{i}$ is $\infty$, the carrier getting permanently trapped and for $\mathrm{p}_{i}=1, t_{i}=0$

We now define

$$
\frac{t_{a}+1}{t_{c}+1}=\frac{p_{c}}{p_{a}}=\frac{1}{R}
$$

A time interval of $\mathrm{N}_{t}$ time steps corresponds to a real time interval of $\mathrm{N}_{t} \tau$ seconds. To calculate $\tau$, we use an estimate of $\tau\left(t_{a}+1\right)$, obtained from NMR linewidth narrowing measurements [13-15]. This is a measure of the average time interval between succesive jumps. Now we can use eqn.(2) to calculate $\xi$, by comparing $\left(\mathrm{j}^{2}>/ 4 t\right)$ from our simulation, with a typical experimental value of $\mathrm{D}[16]$. This gives $\xi=6.14 \mathrm{~A}$ which is physically reasonable being of the order of the interatomic spacing [2]. We now calculate the variation of $\mathrm{D}$ and hence $\sigma$ with the salt fraction $\mathrm{X}$. 


\section{Results and discussion}

Using the above algorithm we have calculated the diffusion coefficients as functions of salt fractions of two polymer electrolytes - $\mathrm{PEO}-\mathrm{NH}_{4} \mathrm{SCN}$ and $\mathrm{PEO}-\mathrm{NH}_{4} \mathrm{I}$. The proportion of crystalline and amorphous sites for the simulation was obtained from experimental estimates of crystallinity from DTA measurements [3,4]. The parameter $\mathrm{R}$ is as given in Table 1 . We find $\mathrm{R}=9.99$ gives satisfactory results in both cases showing that it is a property of the host polymer PEO. The conductivity is estimated using eqn.(4). In eqn.(4) $\mathrm{X}$ are taken from experiment $[3,4]$ and $\mathrm{K}$ is an arbitrary constant as given in Table 1. The calculated values for conductivity fitted well with the experimental values (at room temperature) as shown in Fig.1. and Fig.2. [3,4]. For a pure polymer i.e. zero salt fraction the conductivity according to our model is zero as it should ideally be. However experimental observations indicate $[3,4]$ that the pure polymer has non zero conductivities for reasons mentioned in ref.[2,17]. Our calculation could not be done for any other similar complex due to lack of experimental data of crystallinity (either from XRD or DTA).

In principle our model should be able to predict the optimum salt fraction required to get the highest ionic conductivity for other complexes. The difficulty remains, however, that the experimental crystallinity has been used as input for different salt fractions, in our simulation. It is necessary to predict the variation of crystallinity with salt fraction from a consistent theory or at least emperically to realise the full potential of our model.

The variation of crystallinity with $\mathrm{X}$ appears to be an interesting and complex problem in itself, since available results show peculiar irregularities and are often mutually contradicting[5]. We are at present working on this problem and expect to report our findings later. Some further shortcomings of our model are as follows. The effect of correlation of chain movements was not taken into account. Also from optical micrographs and x-ray difraction analysis it is usually found that the crystalline regions are in the form of spherullites [3]. Based on this, a better picture in the model would have been clustered groups of crystalline sites rather than a random distribution of single crystalline sites. However, in view of the dynamic disorder, our model appears realistic, because a single walker (i.e. a charge carrier) does not see the whole structure, but sees a small locality. 
A useful extension of this model will be to incorporate variation of the renewal time, the time in which the structure rearranges itself. In its present form the renewal time is the shortest possible i.e. one time unit $\tau$.

\section{Acknowledgement :}

One of the authors (AJB) wishes to thank the UGC for providing financial support in the form of a Senior Research Fellowship. The authors are also grateful to Dr. T.K. Ballabh for useful suggestions regarding computer simulation. 


\section{References}

1. M.A. Ratner and D.F. Shriver, Chem. Rev., 88,109 (1988) and references therein.

2. C.A. Vincent, Prog. Solid State Chem., 17, 145 (1987) and references therein.

3. K.K. Maurya, N. Srivastava, S.A. Hashmi and S. Chandra, Jour. of Mater. Sci., 27, 6357 (1992).

4. N. Srivastava, A. Chandra and S. Chandra, Phys. Rev B 52, 225 (1995).

5. D. Prusnikowska, W. Wieczorec, H. Wycislik, M. Siekerski and J. Przyluski, Solid State Ionics, 72, 152 (1994).

6. J.P. Bouchaud and A. Georges, Phys. Rep., 195, 127 (1990).

7. S. Havlin and D. Ben-Avraham, Adv. in Phys., 36, 695 (1987).

8. J.W. Haus and K.W. Kehr, Phys. Rep., 150, 263 (1987).

9. J.F. McCarthy, J. Phys. A, 26, 315 (1986).

10. S.D. Druger, A. Nitzan and M.A. Ratner, J. Chem. Phys., 79, 3133 (1983).

11. S.D. Druger, M.A. Ratner and A. Nitzan, Phys. Rev. B, 31, 3939 (1985).

12. Aninda Jiban Bhattacharyya and S. Tarafdar, to be published.

13. J.B. Boyce and B.A. Huberman, Phys. Rep., 51, 189 (1979).

14. S.H. Chung, K.R. Jeffrey and J.R. Stevens, J. Chem. Phys., 93, 1803 (1991).

15. C. Wang, Q. Liu, Q. Cao, Q. Meng and L. Yang, Solid State Ionics, 53-56, 1106 (1992).

16. W. Gorecki, R. Andreani, C. Berthier, M. Armand, M. Mali, J. Roos and D. Brinkmann, Solid State Ionics, 18-19, 295 (1986).

17. S. Chandra, S.A. Hashmi and G. Prasad, Solid State Ionics, 40-41, 651 (1990). 
Table-1 :Input parameters for calculation of theoretical conductivity and jump distance.

\begin{tabular}{|c|c|c|c|c|c|c|}
\hline Material & K & $\mathrm{p}_{a}$ & $\mathrm{p}_{c}$ & $\mathrm{R}=\mathrm{p}_{a} / \mathrm{p}_{c}$ & $\tau\left(t_{a}+1\right) \mathrm{sec}$ & $\mathrm{D} \mathrm{cm}^{2} / \mathrm{sec}$ \\
\hline $\mathrm{PEO}-\mathrm{NH}_{4} \mathrm{SCN}$ & $2.5 \times \times 10^{-4}$ & 0.999 & 0.1 & 9.99 & $7 \times 10^{-7}$ & $6 \times 10^{-10}$ \\
\hline $\mathrm{PEO}-\mathrm{NH}_{4} \mathrm{I}$ & $1 \times 10^{-3}$ & 0.999 & 0.1 & 9.99 & $7 \times 10^{-7}$ & $6 \times 10^{-10}$ \\
\hline
\end{tabular}




\section{Figure captions :}

1. Figure-1 Plot of theoretical conductivity versus salt fraction for PEO-NH $4 \mathrm{SCN}$. $(\diamond)$ show experimental results.[4]

2. Figure-2 Plot of theoretical conductivity versus salt fraction for PEO-NH 4 I. $(\diamond)$ show experimental results [3]. 
Figure-1

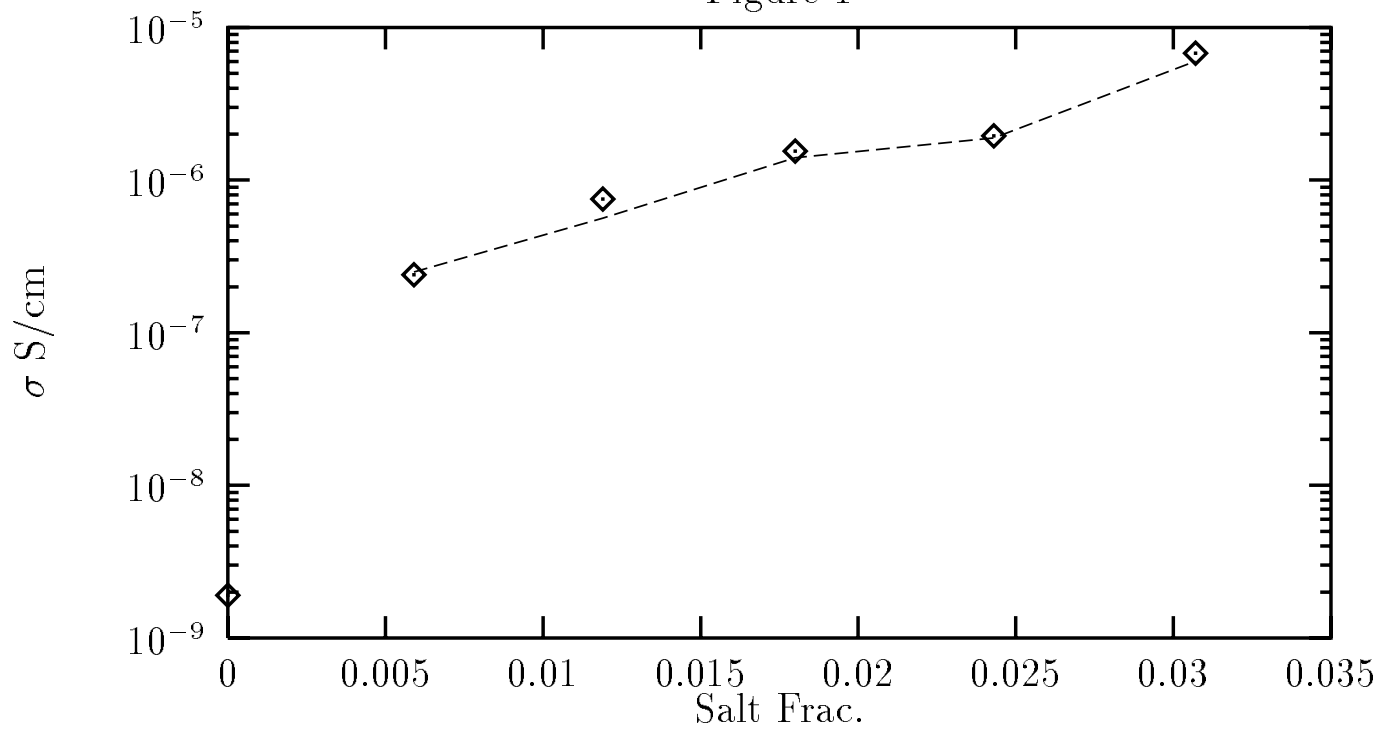


Figure-2

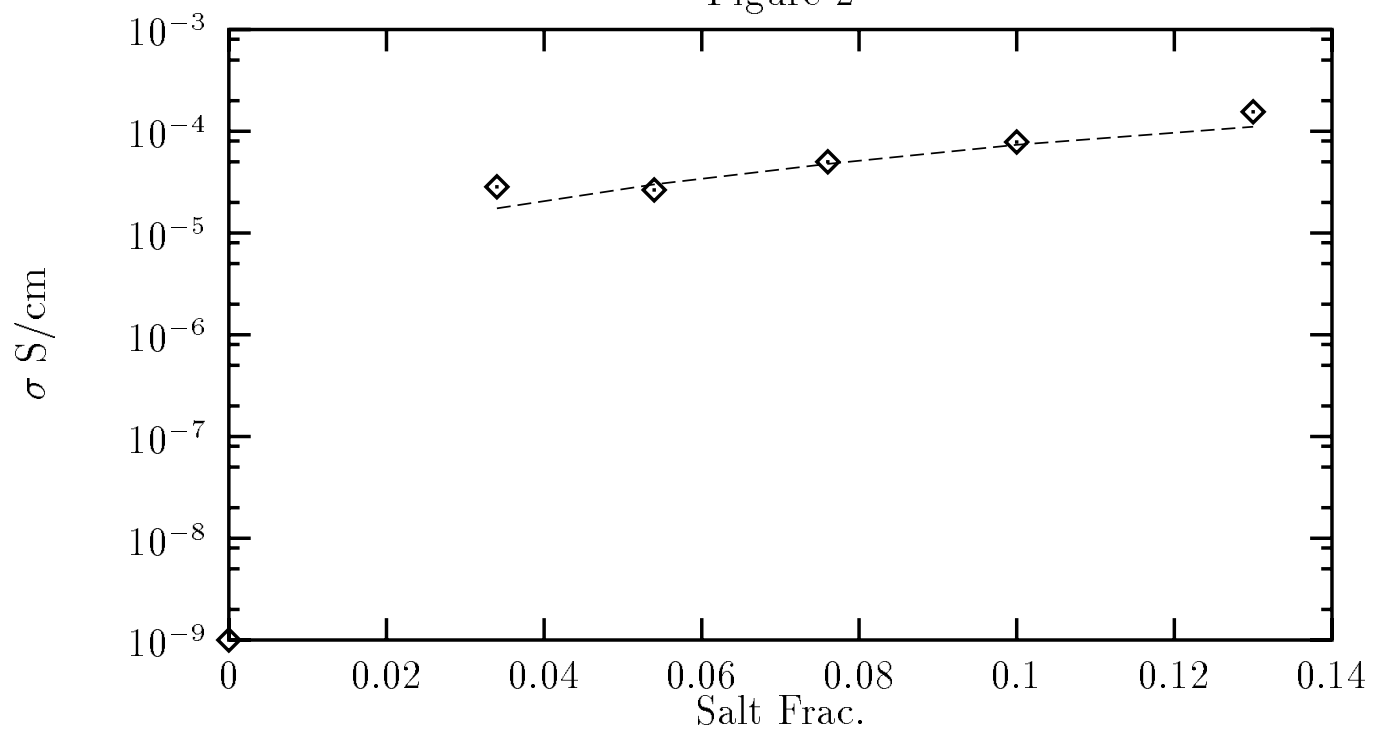

